\title{
Moving beyond Ordinary Factor Analysis in Studies of Personality and Personality Disorder: A Computational Modeling Perspective
}

\author{
Nathaniel Haines Theodore P. Beauchaine \\ Department of Psychology, The Ohio State University, Columbus, OH, USA
}

\section{Keywords}

Personality · Personality disorders · Factor analysis .

Bayesian modeling $\cdot$ Computational modeling

\begin{abstract}
Almost all forms of psychopathology, including personality disorders, are arrived at through complex interactions among neurobiological vulnerabilities and environmental risk factors across development. Yet despite increasing recognition of etiological complexity, psychopathology research is still dominated by searches for large main effects causes. This derives in part from reliance on traditional inferential methods, including ordinary factor analysis, regression, ANCOVA, and other techniques that use statistical partialing to isolate unique effects. In principle, some of these methods can accommodate etiological complexity, yet as typically applied they are insensitive to interactive functional dependencies (modulating effects) among etiological influences. Here, we use our developmental model of antisocial and borderline traits to illustrate challenges faced when modeling complex etiological mechanisms of psychopathology. We then consider how computational models, which are rarely used in the personality disorders literature, remedy some of these challenges when combined with hierarchical Bayesian analysis.

(c) 2020 S. Karger AG, Basel
\end{abstract}

(C) 2020 S. Karger AG, Basel

www.karger.com/psp

Karger

\section{Introduction}

Studying interactions among contributing vulnerabilities to and environmental risk factors for psychopathology across development is essential if we wish to understand etiopathophysiology - defined as complex ontogenic processes through which psychopathology is shaped and maintained over time [1]. Specifying etiopathophysiology is vital because it provides mechanistic targets for prevention and early intervention. This is particularly important for personality disorders (PDs), which are often difficult to treat once established [2]. Traditionally, however, studies of personality disturbance have been confined to those ages 18 years and older given proscriptions against diagnosing PDs among youth [3]. Thus, our understanding of PD development lags somewhat behind our understanding of many other psychiatric disorders.

Fortunately, this is changing [4]. Research conducted over the past 2 decades shows that PDs can be measured reliably in adolescence [5], that adult PDs are almost always preceded by personality disturbance at younger ages [6], and that neural correlates of at least some PDs emerge well before adulthood [7]. This suggests that continued studies of PD development should be pursued. In our work, we study development of both antisocial PD (ASPD) and borderline PD (BPD), which share common 
genetic, temperamental, neural, and environmental risk factors/vulnerabilities $[6,8,9]$. Details of our developmental model can be found in papers cited herein. We focus here on limitations of traditional inferential methods - at least as typically applied - for studying complex psychiatric disorders including ASPD and BPD. We then describe how computational approaches offer certain advantages in modeling personality and $\mathrm{PD}$.

\section{Current Models of PD: Strengths and Limitations}

The prevailing model of PDs described in the DSM-5 [3] carries forward a typology that has been revised very little since it first appeared in the DSM-III 40 years ago [10]. This explicitly categorical model parses PDs into three clusters: Cluster A (odd, eccentric, e.g., paranoid PD), Cluster B (dramatic, erratic, e.g., ASPD, BPD), and Cluster C (anxious, fearful, e.g., avoidant PD). As reviewed by our group and others, including several contributors to this special issue, this rationally derived, discrete model suffers from significant limitations [6]. For purposes of this article, the most important of such limitations concern validity: neither the DSM clusters nor the PDs within them map onto the well replicated, empirically derived dimensional latent structure of personality instantiated in the Big 5. This is problematic because overwhelming evidence links personality disturbance to extreme expressions of Big 5 dimensions [11, 12]. Poor temporal stability of categorical PD diagnoses and high rates of concurrent comorbidity within and across clusters also raise validity concerns $[6,13]$.

In response to these and other limitations, newer models of PDs have emerged that map personality disturbance onto a limited number of empirically derived dimensional traits [14]. The most straightforward of these approaches assess Big 5 personality traits and closely related constructs, which can become maladaptive - both alone and in combinations - at their low and high extremes $[15,16]$. In contrast to the rational approach to PDs exemplified in the DSM, these models are empirically derived using factor analysis (FA), which reduces vast amounts of data into a single model that parsimoniously characterizes patterns of covariance among indicators of personality and personality disturbance.

A somewhat different approach to assessing and diagnosing PDs is used in the DSM-5 alternative model for further study [17]. Using this hybrid model, general degree of personality impairment is first assessed in terms of identity, self-direction, empathy, and intimacy (Crite- rion A). Next, specific pathological personality dysfunction is assessed across 5 trait domains, including negative affectivity, detachment, antagonism, disinhibition, and psychoticism (Criterion B). Indicators of trait domains comprise 25 trait facets (e.g., hostility, grandiosity, impulsivity). Criteria $C$ through $G$ assess issues of differential diagnosis, stability, and sociocultural environment [18].

Notably, all of the approaches summarized thus far are purely symptom based. These models are largely agnostic regarding neurobiological correlates and substrates of personality and PDs. Following a largely separate intellectual tradition are models that link personality traits to neural systems subserving functional domains of human behavior (e.g., approach, avoidance, self-regulation). According to such models, individual differences in activity and reactivity of core neural systems implicated in these behavioral functions are expressed in personality. A straightforward example is reinforcement sensitivity theory (RST), which describes how neural systems of approach and avoidance interact to confer behavioral predispositions including temperament and personality [19]. RST specifies how neural mechanisms of approach, expressed behaviorally as trait impulsivity at one functional extreme, interact with neural systems of avoidance, expressed behaviorally as trait anxiety at one functional extreme.

Approaches such as RST offer two major advantages over exclusively symptom-based models. First, they provide theoretical accounts of etiopathophysiology, the importance of which is outlined above. Second, when combined with appropriate statistical models, they describe interactive dependencies among functional domains of behavior. This refers to situations in which one neural system modulates effects of another on behavior. For example, RST specifies how neural networks subserving trait anxiety dampen behavioral effects of neural networks subserving trait impulsivity [20]. We elaborate on these networks below.

Functional dependencies between neural systems help explain different presentations of externalizing behaviors among children, adolescents, and adults who score equivalently high on trait impulsivity, but low versus high on trait anxiety. Whereas low trait anxiety among those with externalizing disorders is associated with callous-unemotional traits, psychopathy, and especially poor long-term comportment outcomes, high trait anxiety predicts better responses to behavioral treatments, less physical aggression, more positive relationships with peers, and fewer police contacts over time $[21,22]$.

It is important to note that modulating the effects of neurobiological systems on one another are far more 
common than once thought $[1,23]$. This helps explain why functions of single neural systems shows almost no 1:1 correspondences with behavior, including PDs [24]. Such interactive functional dependencies are not captured well by traditional linear models that assume behavior arises from single main effects [25]. In the sections that follow, we describe: (a) our theory of ASPD and BPD development, which includes functional dependencies among neural systems of approach, avoidance, and selfregulation, and (b) how computational modeling can be used to capture such functional dependencies.

\section{An Ontogenic Process Perspective on PDs}

Ontogenesis refers to complex interactions through which biological and environmental influences shape development across the lifespan. Through such processes, biological predispositions can elicit environmental risk exposures through self-selective and evocative effects, and environmental risk exposures can alter neurodevelopment. Our ontogenic process model of ASPD and BPD specifies: (1) neural substrates of trait impulsivity, trait anxiety, and emotion dysregulation; (2) how functional compromises in these neural systems confer vulnerability to psychopathology; and (3) why functional dependencies among these neural systems must be considered to understand etiopathophysiology. Although full articulation of our model is beyond the scope of this article, brief description is warranted. Readers are referred elsewhere for comprehensive accounts $[8,9,21,26]$.

\section{Trait Impulsivity}

We define trait impulsivity as a strong preference for immediate over delayed rewards, difficulties inhibiting intemperate behaviors, and failures to consider potential consequences of immediate actions [27]. Such behaviors characterize both ASPD and BPD $[9,21]$. Although trait impulsivity is highly heritable [28], it is shaped considerably across development by environmental risk exposures [29]. This helps explain why impulsive young boys who incur adversity are vulnerable to ASPD development in later life [21], and why impulsive girls who incur adversity are vulnerable to BPD development in later life [2]. A corollary of such findings is that, in protective environments, progression of impulsivity to PDs is far less likely [9].

Neurally, trait impulsivity derives in part from low tonic activity and low phasic reactivity of the mesolimbic dopamine (DA) system, including the ventral tegmental area and its feed-forward projections to the striatum [27, 30]. This neural network is often referred to as the "primary reward system" or the "pleasure center" of the brain, and is activated when we engage in or anticipate engaging in appetitive behaviors. Low tonic activity and low phasic reactivity in this system imbue an emotional state characterized by anhedonia and irritability $[1,16]$. Anhedonia and irritability are aversive, and therefore motivate reward-seeking, impulsive behaviors, which temporarily upregulate mood [31]. This theory of impulsivity derived originally from invasive research with animals [32] - is supported by extensive neuroimaging work revealing blunted striatal responding in anticipation of incentives among those with ADHD, ASPD, and borderline traits $[30,33,34]$. Notably, when prescribed at therapeutic doses, methylphenidate and other stimulants increase tonic and phasic neural firing in the mesolimbic DA system, elevate mood by relieving anhedonia and irritability, and reduce impulsive, reward-seeking behaviors [35].

\section{Trait Anxiety}

As outlined above, low trait anxiety is a definitional feature of psychopathy and callous-unemotional traits, which are observed among some but not all individuals with ASPD and other externalizing disorders. The complex, interconnected network of brain regions that subserve anxiety, including the hippocampus, periaqueductal gray, medial hypothalamus, amygdala, and broader septohippocampal system were also specified initially through painstaking invasive research with animals [36]. According to RST [19], this network modulates ongoing behaviors when cues in the environment conflict in motivational valence (approach-approach, approach-avoidance, or avoidance-avoidance). Suppressing ongoing behaviors in these situations allows cognitive processes to better arbitrate between risky choices.

We have already described how externalizing children and adolescents who experience anxiety show better long-term outcomes across numerous functional domains than those who experience very little anxiety [21, 22]. Among children with ADHD, anxiety also predicts more deliberative responding on laboratory measures of impulsivity, such as response inhibition [37]. Furthermore, experimental inductions of anxiety attenuate neural responses to reward [38], and comorbid anxiety among externalizing youth is associated with less severe structural compromises in brain regions related to impulsive decision-making [39]. All of these observations suggest interactive functional dependencies between impulsivity and anxiety in affecting behavior. 


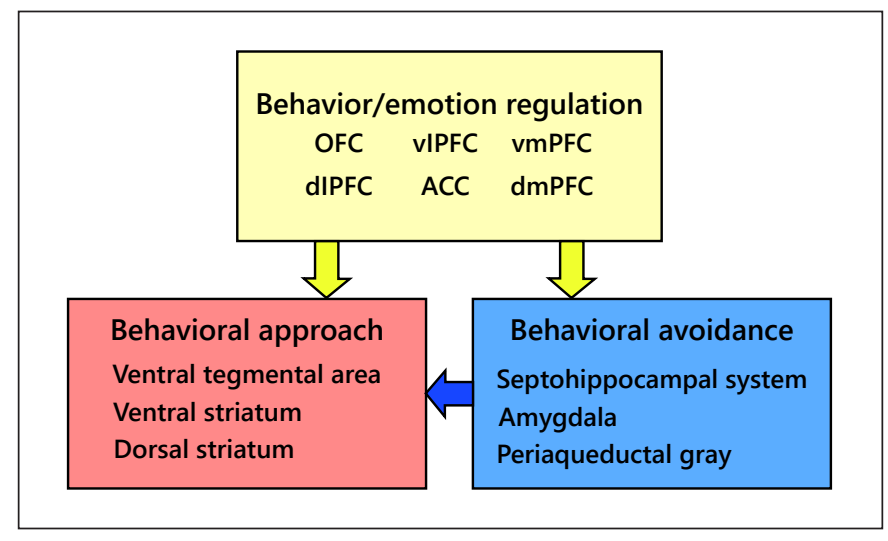

Fig. 1. A heuristic depiction of functional dependencies among neural systems of approach, avoidance, and emotion regulation in affecting impulsive behavior. Impulsivity can arise from excessive approach, deficient avoidance, deficient emotion/self-regulation, or any combination thereof. As a result, disentangling neural sources of impulsivity for any individual may be impossible when assessing behavioral symptoms alone. Furthermore, linear models provide limited insight into such sources. OFC, orbitofrontal cortex; dlPFC, dorsolateral PFC; vlPFC, ventrolateral PFC; ACC, anterior cingulate cortex; vmPFC, ventromedial PFC; dmPFC, dorsomedial PFC.

\section{Emotion Dysregulation}

Emotion dysregulation refers to patterns of emotional experience and/or expression that interfere with effective goal-directed behavior [40, 41]. A burgeoning literature links emotion dysregulation to poor top-down control of subcortical neural systems by functional subdivisions of the prefrontal cortex (PFC). Volitional regulation of impulsivity is supported by orbitofrontal and dorsolateral prefrontal inhibition of mesolimbic activity and reactivity, whereas volitional regulation of anxiety is supported by lateral prefrontal inhibition of amygdalar activity and reactivity [42, 43]. Impulse control and anxiety disorders are characterized by disrupted cortical-subcortical connectivity in these respective neural networks $[44,45]$. Emotion dysregulation therefore derives at least in part from failures in top-down control over subcortical brain regions that generate approach (e.g., wanting, enthusiasm) and avoidance (e.g., apprehension, worry) emotions [46]. Of note, implicated frontal structures that provide top-down regulation of emotion overlap both structurally and functionally with those that subserve executive function and self-control [47].

Among typically developing children and adolescents, subcortical structures, including the striatum and amygdala, reach adult volumetric maturity by age 5 years, whereas structural development of the PFC continues into early adulthood [48]. Both adults with ASPD and
BPD, and adolescents with antisocial and borderline tendencies, exhibit compromised cortical neuromaturation compared with controls $[7,49]$. Taken together, the findings summarized in this section suggest that interactive functional dependencies among subcortical neural systems of approach and avoidance, and cortical neural systems of emotion regulation must be considered in specifying the etiopathophysiology of ASPD and BPD $[8,26]$. Because these neural systems modulate one another, linear methods that evaluate effects of each neural system, one at a time, on behavior/personality, are limited in their capacity to model pathophysiology $[1,16]$. Indeed, impulsivity, an apparently single phenotype, can arise from deficient reward processing, deficient trait anxiety, poor emotion regulation, or any combination thereof (Fig. 1). The specific source or specific sources of impulsivity are therefore difficult if not impossible to infer from confirmatory factor models based on symptoms alone $[25,26]$.

\section{Limits of Ordinary FA in Modeling Functional Dependencies}

Ordinary FA uses simultaneous multiple regression to examine complex patterns of partial correlations between and among manifest indicators (behaviors) and latent constructs (personality traits). Without question, FA has advanced our understanding of PDs, yet as typically applied, it is agnostic regarding complex etiopathophysiological mechanisms of personality and does not accommodate interactive functional dependencies among such mechanisms. Instead, it yields conceptual theories that, although valuable, must be followed by mechanistic research to confirm/disconfirm those theories. In short, ordinary FA tells us which variables sort together; it does tell us why they sort together. It is largely descriptive and therefore of limited explanatory power in isolation [50]. Although additional limitations of FA for making inferences about psychopathology have been described [51], they are not relevant to the argument at hand and are therefore not considered.

\section{Modeling Behavior across Timescales and Levels of Analysis}

As outlined above, development of psychopathology - including PDs - is inherently complex $[1,24]$. In the sections that follow, we provide a brief, highly focused overview of conceptual and statistical frameworks that more effectively model such complexity across time and 


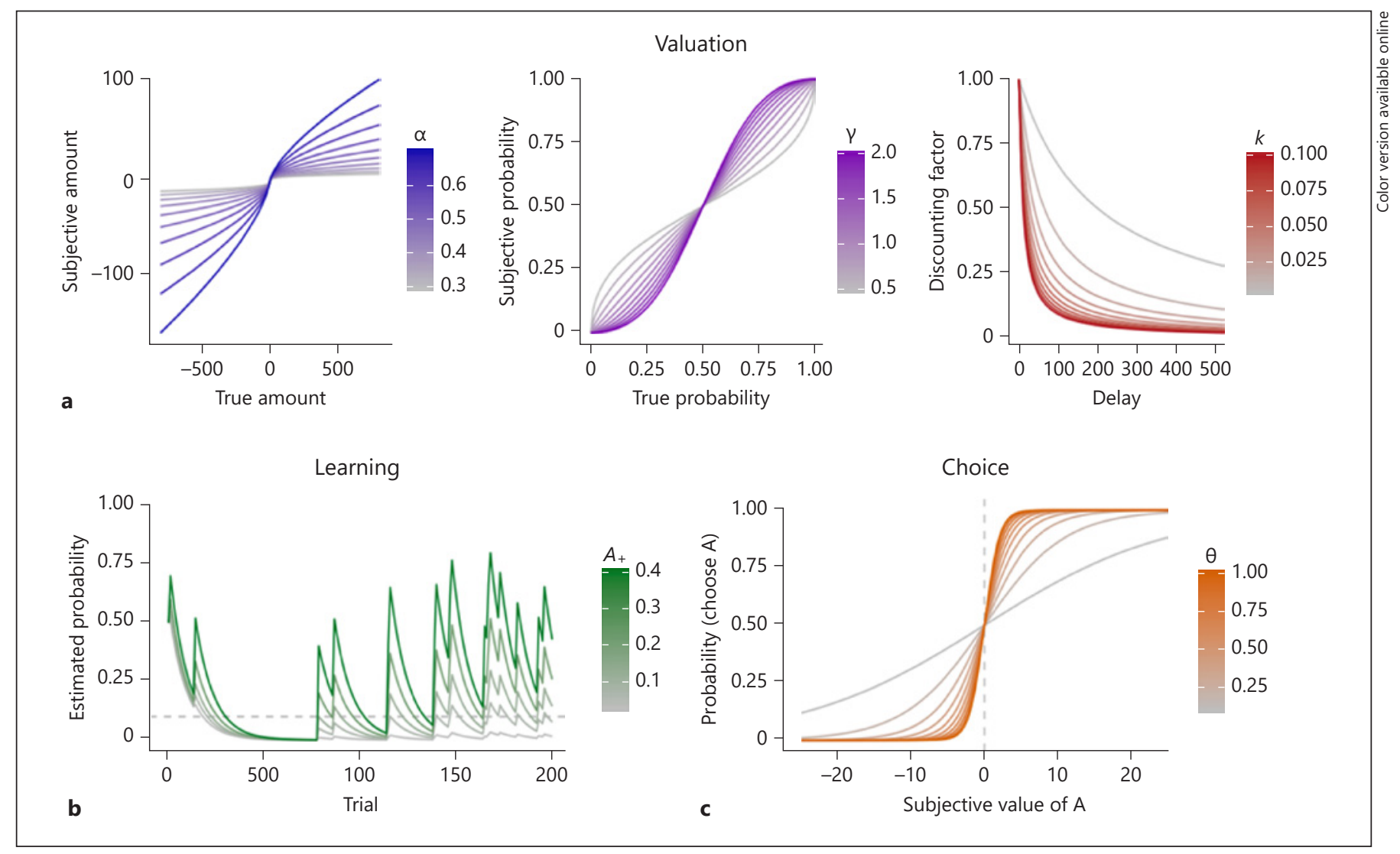

Fig. 2. a Valuation mechanisms offer formal representations that capture how individuals assign subjective value to different dimensions of a given decision. Here, we depict commonly used functions that capture effects of diminishing sensitivity to magnitude (using a prospect theory [81] utility function where $\alpha$ captures reinforcer/ punishment sensitivity and $\lambda$ [loss aversion] is set to 1.5 , indicating more sensitivity to losses than gains), probability weighting (using a function that captures overweighting or underweighting of low or high probabilities [82]), and delay discounting of reinforcers/punishments (using a common hyperbolic discounting function [83]). b Learning mechanisms can capture changes in behavior over time due to experience. Here, we show learning curves for a model estimating the probability of a rare event (indicated by the dashed gray line, with a true probability of 0.10 ). The model updates at different rates for positive and negative prediction errors (i.e., when out-

levels of analysis. Our goal is to introduce readers to these models and their conceptual bases. Given space constraints, we cannot provide full descriptions or tutorials. Interested readers are referred to sources cited herein for more complete descriptions.

\section{Modeling and Identifying Functional Dependencies in Impulsive Decision-Making}

Several approaches accommodate modeling multiple interactive determinants of individual-level impulsive comes are better or worse than expected), where the positive learning rate $\left(\mathrm{A}_{+}\right)$is varied and the negative learning rate $\left(A_{-}\right)$is set to 0.10 in the example $[62,67]$. Different learning rates explain systematic over- or underestimation of reinforcer/punishment values (probabilities) - a core aspect of behavioral impulsivity. c Choice mechanisms capture how likely individuals are to make specific actions given values assigned to each option/action from previous steps. Here, we use a logistic (softmax) function [65], which shows the probability of choosing option A over option B while varying the subjective value of option $A$ (where the value of option $B$ is always 0 , indicated by the dashed gray line) and a choice consistency parameter $(\theta)$ that captures how deterministically individuals choose the higher-valued option over the lower-valued option. Together, mechanisms from $\mathbf{a}, \mathbf{b}$, and $\mathbf{c}$ can be integrated to account for a wide range of both adaptive and maladaptive behaviors.

behaviors. Many of these approaches overlap despite having different names in the literature. Examples include value-based decision-making/decision theory [52], model-based cognitive neuroscience [53], and computational psychiatry/computational phenotyping $[54,55]$. Computational models from these research areas identify distinct contributing mechanisms of behavior, including how individuals represent, valuate, learn from, and choose among actions in response to potential rewards and punishments (non-reward). Individual differ- 
ences in such predispositions contribute to personality and PD [56].

An often underappreciated but important difference between traditional approaches such as ordinary FA and its extensions [57] versus computational modeling is that confirmatory FA models - at least as typically applied are agnostic regarding underlying mechanisms of behavior [51]. Instead, they are applied primarily to behavioral data (e.g., self-report items, summary statistics of behavioral measures, etc.) to quantify patterns of covariance among observed variables - with no assumptions regarding the underlying mechanisms of those behaviors [58] (e.g., cognitive, physiological, neural; for a conceptual exception, see Beauchaine and Zisner [26]). Although Bayesian FA exists and can accommodate nested hierarchies, interactions across levels of analysis, and complex nonlinearities [59], it has also been applied agnostically with respect to underlying neural, cognitive, and physiological mechanisms of behavior.

In contrast, the computational models we describe: (a) are based on explicit mathematical functions that model either known or theoretically derived mechanisms of behavior (Fig. 2), (b) can be modified to accommodate iteratively improved understanding of mechanistic parameters (e.g., informed likelihoods), which in turn yields more precise predictions of future behavior, and (c) are evaluated holistically based on how well they explain theoretically relevant patterns in observed data as opposed to whether or not they identify statistically significant effects [60-62]. Taken together, these features demonstrate advantages of computational models toward describing complex psychological phenomena and their underlying substrates. Computational models also gravitate away from the notion that behavior can be explained by a small number of large main effects, toward the more realistic view that behavior is multiply determined by functional interactions among cognitive, neural, emotional, and physiological mechanisms that are often context dependent [25]. We are not suggesting that behavior is subordinate to other levels of analysis in our models. On the contrary - behavior is what we seek to explain and understand. Such an explanation requires us to specify mechanisms through which behavior is effected, across all relevant levels of analysis [2, 6, 21, 62].

Work by our group and others shows that computational models of impulsive behavior, including components described in Figure 2, can: (a) differentiate clinical from non-clinical groups, and (b) generate counterintuitive predictions about behavior based on known interactions among mechanisms [63]. In one of the first studies of this kind, for example, Yechiam et al. [64] used computational modeling to demonstrate that those who show non-specific increases in risky decision-making on the Iowa Gambling Task differ at more mechanistic levels. Chronic cannabis and cocaine users showed a bias for gains over losses and "myopia" for immediate over delayed rewards (higher discounting rates of distant outcomes). In contrast, younger, non-chronic substance users showed no such biases. Similar findings have emerged across a range of behavioral tasks among those with externalizing disorders $[65,66]$.

Such models hold potential to "bridge" explanatory gaps between behaviors, neural functions, and traits. For example, reinforcement learning models based on DA signaling capture both differential sensitivity to outcomes that are more positive or negative than expected [67], and relations between reinforcer availability and mood stability/lability [68]. Moreover, computational models of selfcontrol link model parameters to neural dynamics, supporting their use as proxies for neural processes that are otherwise unobservable. For example, Turner et al. [69] developed a computational model of delay discounting that includes a within-subject lateral inhibition parameter (which functions in the model to downregulate impulsive valuation). When fitting the model to behavioral data alone, the lateral inhibition parameter correlated across participants with BOLD signaling in the dorsolateral PFC - a key neural structure implicated in self-control and development of externalizing psychopathology $[46,49]$.

In our own work, we have shown that trait impulsivity and state anxiety interact to predict delay discounting rates (according to a traditional regression model), and that the same interaction can be produced by a more mechanistic computational model that assumes anxiety and impulsivity relate to distinct decision mechanisms of reward amount and delay valuation, respectively. The model therefore provides insight into functional outcomes among impulsive, externalizing adults with comorbid anxiety symptoms, as described above [63]. These and other findings indicate improved prediction of behavior when theoretical mechanisms and their functional dependencies are modeled directly. They also demonstrate how computational modeling can account for complex interactive mechanisms among individual differences across levels of analysis in affecting phenotypically similar and in some cases indistinguishable behaviors.

\section{Modeling Developmental Change}

In the framework described herein, two interrelated types of development must be modeled, including: (1) in- 


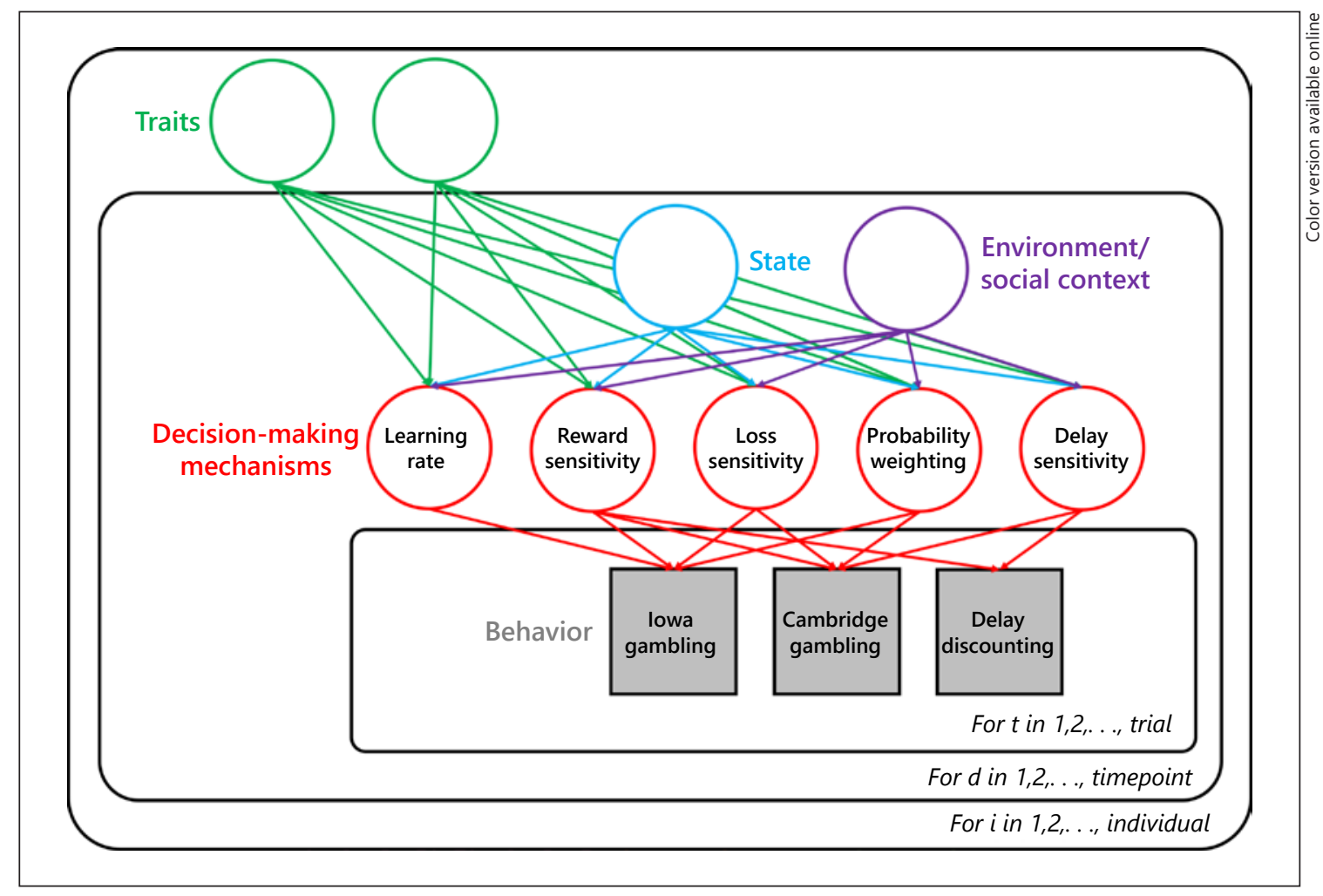

Fig. 3. A simplified graphical representation of how HBA can be used to develop complex models that link levels and timescales of analysis in theoretically motivated ways. Open circles indicate model components with free parameters estimated from data. Gray squares indicate observed behavior (e.g., reaction times to gains vs. losses) in tasks that measure the construct of interest (in this case, tasks often used to measure impulsive behavior). Arrows represent probabilistic relations between variables at different levels. The large rectangles indicate hierarchical nesting (e.g., trials within timepoints within individuals). Note that relations between variables can take any specified form - including simple linear to complex nonlinear. In the model shown, traits such as impulsivity, anxiety, and emotion dysregulation are assumed to exert constant effects on decision-making over time, whereas state influences, as inferred from: (a) physiological, neural, or experimental design data, and/or (b) immediate environmental/

dividual-level maturation in behavioral traits, neurobiological functions, and cognitive processes; and (2) environmental-/social-level changes in types and availabilities of reinforcers over time. For individual-level change, developmental computational psychiatry offers a framework that is well suited to bridge behavioral and neural levels of analysis [70]. For example, computational models of reward learning show that prediction error coding in the striatum grows during childhood, peaks in adolescence, and declines in adulthood [71]. Future research might examine how such findings relate to functional social contexts, are assumed to vary across timepoints. Ideally, each level is comprised of a unique sub-model. For example, state effects (turquoise) may be estimated from a general linear model applied to neuroimaging data (red), and traits from FA (green). Such joint modeling methods can improve the precision of parameter estimates at all levels [53]. Similarly, development could be modeled through estimating changes in traits or environmen$\mathrm{tal} / \mathrm{social}$ contexts across timepoints, as is often done using growth models. Unlike non-Bayesian methods, prior expert information can be used to constrain estimation at all levels in the form of prior distributions, which can greatly improve the quality of inference (particularly in small samples) [84]. Similarly, once relations are identified, we can use posterior simulations to predict how different trait/state/context effects may affect behavior, which can lead to insightful and sometimes counterintuitive theoretical predictions [63].

changes in neural systems implicated in anxiety and selfregulation, and how functional neuromaturation and dependencies among neural systems relate to development of personality and PDs. Learning models also hold potential for evaluating cognitive and neural mechanisms of treatment response [72]. We note here that latent growth curve models, which extend FA to longitudinal data, could in principle be applied to such data. To date, however, few such applications have appeared in the literature. Nevertheless, joint modeling in which highly precise laboratory response parameters (indexed by computa- 
tional models), are "linked" to personality attributes over time (indexed using FA), hold considerable promise (Fig. 3). Furthermore, statistical approaches such as hierarchical Bayesian analysis (HBA; described below) offer unified frameworks within which such models can be developed [53, 59].

Borrowing from computational sociology and epidemiology, environmental and social changes are modeled as interactions among networks of individuals (termed "agents") over time [73]. Including these effects is essential given overwhelming evidence that nonshared environments magnify personality traits across development [16]. Using such a framework, complex nested family effects and broader social dynamics can emerge from simple decision mechanisms/ rules that individuals follow within immediate, proximal, and more distal social networks [74]. Such models are already used to infer etiological factors of disease spread following chemical exposures, and to identify possible contributing factors to spatially correlated disease expressions $[59,75]$.

\section{HBA to Link Levels of Analysis}

Continuing with this discussion, HBA provides a straightforward statistical framework for developing and estimating parameters from complex models that include individual-level, social, and developmental mechanisms (Fig. 3). A principal benefit of HBA over competing approaches is that it simultaneously pools information across all levels of analysis, which: (a) increases the precision of parameter estimates at each level, and (b) allows us to integrate over uncertainty ("error" in frequentist terms) both within and across levels. More importantly, HBA allows parameters in complex computational models to be estimated simultaneously with parameters from more traditional measurement models in individual differences research [76]. For example, with HBA we can fit: (1) a confirmatory factor analytic model to self-report trait measures, (2) a computational model to precise behavioral data (e.g., reaction times, response latencies, etc.), and (3) estimate relations between individual difference estimates from (1) and (2), i.e., correlations between latent traits and computational model parameters - all within a single model. We cannot provide a detailed description of HBA here. Instead, we refer readers to beginning and advanced tutorials [53, 77-80].

For personality/PDs researchers, use of HBA and computational modeling allows us to ask questions that are more difficult and, in some cases, impossible to ad- dress with traditional approaches. For example, once associations between traits and decision mechanisms are identified, we can use these identified estimates to inform prior distributions in future studies using HBA, facilitating accumulation of empirical knowledge and iterating toward more efficient prediction of future behavior. Priors can also be used to simulate effects of changes in traits on future behavior through their influence on decision-making. Because decision mechanisms are shared across many behavioral contexts (e.g., both risky decision-making tasks and delay-discounting tasks require people valuate rewards) and interact in complex yet interpretable ways to affect behavior [53], computational methods are often conducive to more rapid theory development and testing than traditional approaches that seek 1:1 correspondences between traits and behavior.

\section{Discussion}

PDs emerge from complex interactions among biological predispositions and environmental risk factors across development. Traditional statistical models such as confirmatory FA are useful in delineating the latent structure of PDs, but tell us little about underlying mechanisms of behavior. This is especially problematic when apparently single behavioral phenotypes derive from multiple, functionally dependent neural processes. As the field moves toward treatments that target mechanisms of psychopathology directly (sometimes termed precision psychiatry), specifying etiopathophysiology at the individual level will be increasingly important. For example, impulsivity that derives exclusively from deficient mesolimbic DA activity/reactivity will likely respond better to stimulants than impulsivity derived exclusively from poor top-down executive control.

PDs and all behavioral traits are determined by complex interactions among genetically influenced personality attributes, neural and physiological organization and activity, physical and psychological states, social and environmental contexts across development $[6,8,9,21]$. Computational modeling offers a set of methods for interrogating functional dependencies among these complex determinants of behavior, and HBA offers a flexible statistical framework that accommodates such complexity. Although the full promise of computational approaches is yet to be realized, we anticipate rapid progress in the upcoming decades as computational methods become increasingly accessible [77]. 


\section{Statement of Ethics}

No participant data were used for this paper.

\section{Disclosure Statement}

The authors declare no conflicts of interest.

\section{Funding Sources}

Preparation of this article was supported by grants UH2DE025980 and UL1TR002733 from the National Institutes of Health.

\section{Author Contributions}

N.H. and T.P.B drafted the paper. Both authors contributed to the concepts described throughout the paper and approved of the final version.

\section{References}

1 Beauchaine TP, Constantino JN. Redefining the endophenotype concept to accommodate transdiagnostic vulnerabilities and etiological complexity. Biomarkers Med. 2017 Sep;11(9): 769-80.

2 Beauchaine TP, Hinshaw SP, Bridge JA. Nonsuicidal self-injury and suicidal behaviors in girls: the case for targeted prevention in preadolescence. Clin Psychol Sci. 2019 Jul;7(4): 643-67.

3 American Psychiatric Association. Diagnostic and statistical manual of mental disorders. 5th ed. Arlington (VA): APA; 2013.

4 Cicchetti D. Illustrative developmental psychopathology perspectives on precursors and pathways to personality disorder: commentary on the special issue. J Pers Disord. 2014 Feb;28(1):172-9.

5 Miller AL, Muehlenkamp JJ, Jacobson CM. Fact or fiction: diagnosing borderline personality disorder in adolescents. Clin Psychol Rev. 2008 Jul;28(6):969-81.

6 Beauchaine TP, Klein DN, Crowell SE, Derbidge C, Gatzke-Kopp L. Multifinality in the development of personality disorders: a Biology $\mathrm{x}$ Sex $\mathrm{x}$ Environment interaction model of antisocial and borderline traits. Dev Psychopathol. 2009;21(3):735-70.

7 Beauchaine TP, Sauder CL, Derbidge CM, Uyeji LL. Self-injuring adolescent girls exhibit insular cortex volumetric abnormalities that are similar to those seen in adults with borderline personality disorder. Dev Psychopathol. 2019 Oct;31(4):1203-12.

8 Beauchaine TP. A developmental psychopathology perspective on the emergence of antisocial and borderline personality pathologies across the lifespan. In: Lejuez CW, Gratz KL, editors. Handbook of personality disorders. NY: Cambridge University Press; 2020. pp. 94-8.

9 Crowell SE, Beauchaine TP, Linehan MM. A biosocial developmental model of borderline personality: elaborating and extending Linehan's theory. Psychol Bull. 2009 May; 135(3): 495-510.

10 American Psychiatric Association. Diagnostic and statistical manual of mental disorders. 3rd ed. Washington: APA; 1980.
11 Krueger RF, Eaton NR, Clark LA, Watson D, Markon KE, Derringer J, et al. Deriving an empirical structure of personality pathology for DSM-5. J Pers Disord. 2011 Apr;25(2): $170-91$.

12 Trull TJ, Widiger TA. Dimensional models of personality: the five-factor model and the DSM-5. Dialogues Clin Neurosci. 2013 Jun; 15(2):135-46.

13 Durbin CE, Klein DN. Ten-year stability of personality disorders among outpatients with mood disorders. J Abnorm Psychol. 2006 Feb; 115(1):75-84

14 Widiger TA, Costa PT, editors. Personality disorders and the five-factor model of personality. 3rd ed. Washington: American Psychological Association; 2013. https://doi. org/10.1037/13939-000.

15 Bagby RM, Widiger TA. Five Factor Model personality disorder scales: an introduction to a special section on assessment of maladaptive variants of the five factor model. Psychol Assess. 2018 Jan;30(1):1-9.

16 Beauchaine TP, Tackett JL. Irritability as a transdiagnostic vulnerability trait: current issues and future directions. Behav Ther. 2020 Mar;51(2):350-64.

17 Zachar P, Krueger RF, Kendler KS. Personality disorder in DSM-5: an oral history. Psychol Med. 2016 Jan;46(1):1-10.

18 Waugh MH, Hopwood CJ, Krueger RF, Morey LC, Pincus AL, Wright AG. Psychological assessment with the DSM-5 alternative model for personality disorders: tradition and innovation. Prof Psychol Res Pr. 2017 Apr;48(2):79-89.

19 Corr PJ, McNaughton N. Neuroscience and approach/avoidance personality traits: a two stage (valuation-motivation) approach. Neurosci Biobehav Rev. 2012 Nov;36(10):2339-54.

20 Corr PJ, McNaughton N. Neural mechanisms of low trait anxiety and risk for externalizing behavior. In: Beauchaine TP, Hinshaw SP, editors. The Oxford handbook of externalizing spectrum disorders. NY: Oxford University Press; 2016. pp. 220-38.

21 Beauchaine TP, Zisner AR, Sauder CL. Trait impulsivity and the externalizing spectrum. Annu Rev Clin Psychol. 2017 May;13(1):34368.
22 Walker JL, Lahey BB, Russo MF, Frick PJ, Christ MA, McBurnett K, et al. Anxiety, inhibition, and conduct disorder in children: I. Relations to social impairment. J Am Acad Child Adolesc Psychiatry. 1991 Mar;30(2): 187-91.

23 Finucane B, Challman TD, Martin CL, Ledbetter DH. Shift happens: family background influences clinical variability in genetic neurodevelopmental disorders. Genet Med. 2016 Apr;18(4):302-4.

24 Beauchaine TP, Constantino JN, Hayden EP. Psychiatry and developmental psychopathology: unifying themes and future directions. Compr Psychiatry. 2018 Nov;87: $143-52$.

25 Beauchaine TP, Hinshaw SP. RDoC and psychopathology among youth: misplaced assumptions and an agenda for future research. J Clin Child Adolesc Psychol. 2020 June; 49(3):322-40.

26 Beauchaine TP, Zisner A. Motivation, emotion regulation, and the latent structure of psychopathology: an integrative and convergent historical perspective. Int J Psychophysiol. 2017 Sep;119:108-18.

27 Sagvolden T, Johansen EB, Aase H, Russell VA. A dynamic developmental theory of attention-deficit/hyperactivity disorder (ADHD) predominantly hyperactive/impulsive and combined subtypes. Behav Brain Sci. 2005;28(3):397-419.

28 Dhamija D, Tuvblad C, Baker LA. Behavioral genetics of the externalizing spectrum. In: Beauchaine TP, Hinshaw SP, editors. The Oxford handbook of externalizing spectrum disorders. NY: Oxford University Press; 2016. pp. 105-24

29 Burt SA. Rethinking environmental contributions to child and adolescent psychopathology: a meta-analysis of shared environmental influences. Psychol Bull. 2009 Jul;135(4):60837.

30 Plichta MM, Scheres A. Ventral-striatal responsiveness during reward anticipation in ADHD and its relation to trait impulsivity in the healthy population: a meta-analytic review of the fMRI literature. Neurosci Biobehav Rev. 2014 Jan;38:125-34. 
31 Zisner A, Beauchaine TP. Neural substrates of trait impulsivity, anhedonia, and irritability: mechanisms of heterotypic comorbidity between externalizing disorders and unipolar depression. Dev Psychopathol. 2016 Nov; 28(4pt1):1177-208.

32 Milner PM. Brain-stimulation reward: a review. Can J Psychol. 1991 Mar;45(1):1-36.

33 Murray L, Shaw DS, Forbes EE, Hyde LW. Reward-related neural correlates of antisocial behavior and callous-unemotional traits in young men. Biol Psychiatry Cogn Neurosci Neuroimaging. 2017 May;2(4): 346-54.

34 Sauder CL, Derbidge CM, Beauchaine TP. Neural responses to monetary incentives among self-injuring adolescent girls. Dev Psychopathol. 2016 Feb;28(1):277-91.

35 Rubia K, Halari R, Cubillo A, Mohammad AM, Brammer M, Taylor E. Methylphenidate normalises activation and functional connectivity deficits in attention and motivation networks in medication-naïve children with ADHD during a rewarded continuous performance task. Neuropharmacology. 2009 Dec; 57(7-8):640-52.

36 Gray JA, McNaughton N. The neuropsychology of anxiety: An enquiry into the functions of the septo-hippocampal system. 2nd ed. Oxford, UK: Oxford University Press; 2000.

37 Bloemsma JM, Boer F, Arnold R, Banaschewski T, Faraone SV, Buitelaar JK, et al. Comorbid anxiety and neurocognitive dysfunctions in children with ADHD. Eur Child Adolesc Psychiatry. 2013 Apr;22(4):225-34.

38 Engelmann JB, Meyer F, Fehr E, Ruff CC. Anticipatory anxiety disrupts neural valuation during risky choice. J Neurosci. 2015 Feb; 35(7):3085-99.

39 Sauder CL, Beauchaine TP, Gatzke-Kopp LM, Shannon KE, Aylward E. Neuroanatomical correlates of heterotypic comorbidity in externalizing male adolescents. J Clin Child Adolesc Psychol. 2012;41(3):346-52.

40 Beauchaine TP, Cicchetti D. Emotion dysregulation and emerging psychopathology: A transdiagnostic, transdisciplinary perspective. Dev Psychopathol. 2019 Aug;31(3):799804.

41 Beauchaine TP, Crowell SE, editors. The Oxford handbook of emotion dysregulation. New York: Oxford University Press; 2020. https://doi.org/10.1093/oxford$\mathrm{hb} / 9780190689285.001 .0001$.

42 Heatherton TF. Neuroscience of self and selfregulation. Annu Rev Psychol. 2011;62(1): 363-90.

43 Tone EB, Garn CL, Pine DS. Anxiety regulation: A developmental psychopathology perspective. In: Cicchetti D, editor. Developmental neuroscience. Developmental psychopathology. Volume 2. 3rd ed. Hoboken (NJ): Wiley; 2016. pp. 523-56.

44 Gold AL, Shechner T, Farber MJ, Spiro CN, Leibenluft E, Pine DS, et al. Amygdala-cortical connectivity: associations with anxiety, development, and threat. Depress Anxiety. 2016 Oct;33(10):917-26.

45 Shannon KE, Sauder C, Beauchaine TP, Gatzke-Kopp LM. Disrupted effective connectivity between the medial frontal cortex and the caudate in adolescent boys with externalizing behavior disorders. Crim Justice Behav. 2009; 36(11):1141-57.

46 Beauchaine TP. Future directions in emotion dysregulation and youth psychopathology. J Clin Child Adolesc Psychol. 2015;44(5):87596.

47 Zelazo PD. Executive function: reflection, iterative reprocessing, complexity, and the developing brain. Dev Rev. 2015;38:55-68.

48 Brain Development Cooperative Group. Total and regional brain volumes in a population-based normative sample from 4 to 18 years: the NIH MRI Study of Normal Brain Development. Cereb Cortex. 2012 Jan;22(1): $1-12$.

49 De Brito SA, Mechelli A, Wilke M, Laurens KR, Jones AP, Barker GJ, et al. Size matters: increased grey matter in boys with conduct problems and callous-unemotional traits. Brain. 2009 Apr;132(Pt 4):843-52.

50 McDonough-Caplan H, Klein DN, Beauchaine TP. Comorbidity and continuity of depression and conduct problems from elementary school to adolescence. J Abnorm Psychol. 2018 Apr;127(3):326-37.

51 Burns GL, Geiser C, Servera M, Becker SP, Beauchaine TP. Application of the bifactor S-1 model to multisource ratings of ADHD/ ODD symptoms: an appropriate bifactor model for symptom ratings. J Abn Child Psychol. 2020;48(7):881-94.

52 Doya K. Modulators of decision making. Nat Neurosci. 2008 Apr;11(4):410-6.

53 Turner BM, Forstmann BU, Love BC, Palmeri TJ, Van Maanen L. Approaches to analysis in model-based cognitive neuroscience. J Math Psychol. 2017 Feb;76B:65-79.

54 Wiecki TV, Poland J, Frank MJ. Modelbased cognitive neuroscience approaches to computational psychiatry: clustering and classification. Clin Psychol Sci. 2015;3(3): 378-99.

55 Patzelt EH, Hartley CA, Gershman SJ. Computational phenotyping: using models to understand individual differences in personality, development, and mental illness. Personal Neurosci. 2018 Oct;1:e18.

56 Hirsh JB, Morisano D, Peterson JB. Delay discounting: interactions between personality and cognitive ability. J Res Pers. 2008;42(6): 1646-50.

57 Bollen KA, Curran PJ. Latent curve models: A structural equation perspective. Hoboken (NJ): Wiley; 2006.

58 Hampson SE. Personality processes: mechanisms by which personality traits "get outside the skin”. Annu Rev Psychol. 2012;63(1):31539.

59 Ferrari F, Dunson D. Bayesian factor analysis for inference on interactions. J Am Stat Assoc. 2020;1-12.; advance online pub.
60 Navarro DJ. Between the devil and the deep blue sea: tensions between scientific judgement and statistical model selection. Comput Brain Behav. 2019;2(1):28-34.

61 Kellen D. A model hierarchy for psychological science. Comput Brain Behav. 2019;2(34):160-5.

62 Wilson RC, Collins AG. Ten simple rules for the computational modeling of behavioral data. eLife. 2019;8:e49547.

63 Haines N, Beauchaine TP, Galdo M et al. Anxiety modulates preference for immediate rewards in trait-impulsive individuals: a hierarchical Bayesian analysis. Clin Psychol Sci. Forthcoming 2020.

64 Yechiam E, Busemeyer JR, Stout JC, Bechara A. Using cognitive models to map relations between neuropsychological disorders and human decision-making deficits. Psychol Sci. 2005 Dec;16(12):973-8.

65 Haines N, Vassileva J, Ahn WY. The outcome-representation learning model: a novel reinforcement learning model of the Iowa Gambling Task. Cogn Sci. 2018 Nov;42(8): 2534-61.

66 Romeu RJ, Haines N, Ahn WY, Busemeyer JR, Vassileva J. A computational model of the Cambridge gambling task with applications to substance use disorders. Drug Alcohol Depend. 2020 Jan;206:107711.

67 Daw ND, Kakade S, Dayan P. Opponent interactions between serotonin and dopamine. Neural Netw. 2002 Jun-Jul;15(4-6): 603-16.

68 Eldar E, Rutledge RB, Dolan RJ, Niv Y. Mood as representation of momentum. Trends Cogn Sci. 2016 Jan;20(1):15-24.

69 Turner BM, Rodriguez CA, Liu Q, Molloy MF, Hoogendijk M, McClure SM. On the neural and mechanistic bases of self-control. Cereb Cortex. 2019 Feb;29(2):732-50.

70 Hauser TU, Will GJ, Dubois M, Dolan RJ. Annual Research Review: developmental computational psychiatry. J Child Psychol Psychiatry. 2019 Apr;60(4):412-26.

71 Cohen JR, Asarnow RF, Sabb FW, Bilder RM, Bookheimer SY, Knowlton BJ, et al. A unique adolescent response to reward prediction errors. Nat Neurosci. 2010 Jun; 13(6):669-71.

72 Moutoussis M, Shahar N, Hauser TU, Dolan RJ. Computation in psychotherapy, or how computational psychiatry can aid learningbased psychological therapies. Comput Psychiatr. 2018 Feb;2:50-73.

73 Macy MW, Willer R. From factors to actors: computational sociology and agent-based modeling. Annu Rev Sociol. 2002;28(1):143-66.

74 McElreath R, Lubell M, Richerson PJ, Waring TM, Baum W, Edsten E, et al. Applying evolutionary models to the laboratory study of social learning. Evol Hum Behav. 2005;26(6): 483-508.

75 Mezzetti M. Bayesian factor analysis for spatially correlated data: application to cancer incidence data in Scotland. Stat Methods Appl. 2012;21(1):49-74. 
76 Boehm U, Steingroever H, Wagenmakers EJ. Using Bayesian regression to test hypotheses about relationships between parameters and covariates in cognitive models. Behav Res Methods. 2018 Jun;50(3):1248-69.

77 Ahn WY, Haines N, Zhang L. Revealing neuro-computational mechanisms of reinforcement learning and decision-making with the hBayesDM package. Comput Psychiatr. 2017 Oct;1:24-57.

78 Kruschke JK. Doing Bayesian data analysis. 2nd ed. NY: Academic Press; 2015.
79 McElreath R. Statistical rethinking: A Bayesian course with examples in $\mathrm{R}$ and Stan. Boca Raton (FL): CRC Press; 2016.

80 Lee MD, Wagenmakers EJ. Bayesian cognitive modeling. Cambridge: Cambridge University Press; 2013. https://doi.org/10.1017/ CBO9781139087759.

81 Kahneman D, Tversky A. Prospect theory: an analysis of decision under risk. Econometrica. 1979;47(2):263-92.
82 Goldstein WM, Einhorn HJ. Expression theory and the preference reversal phenomena. Psychol Rev. 1987;94(2):236-54.

83 Mazur JE. An adjustment procedure for studying delayed reinforcement. In: Commons ML Mazur JE, Nevin JA, Rachlin H, editors. Quantitative analyses of behavior. Volume 5. Hillsdale: Erlbaum; 1987. pp. 55-73.

84 Schad DJ, Betancourt M, Vasishth S. Toward a principled Bayesian workflow in cognitive science. Psychol Methods. 2020. doi: 10.1037/ met0000275. 